\title{
Synthesis of chitosan-grafted poly-acrylic acid (CTS-g-PAA) hydrogel and its potential application in biosensors for signal enhancing and bioanalysis
}

\author{
Esmaeel Alipour ${ }^{1, *}$ (D), Sheida Norouzi ${ }^{1}$, Hajar Yousefzadeh ${ }^{1}$, Reza Mohammadi ${ }^{2}$, and \\ Mohammad Sadegh Amini-Faz| ${ }^{3}$ \\ ${ }^{1}$ Electroanalytical Chemistry Laboratory, Department of Analytical Chemistry, Faculty of Chemistry, University of Tabriz, Tabriz, \\ Iran \\ ${ }^{2}$ Polymer Research Laboratory, Department of Organic and Biochemistry, Faculty of Chemistry, University of Tabriz, Tabriz, Iran \\ ${ }^{3}$ Research Laboratory of Advanced Polymer Material, Department of Applied Chemistry, Faculty of Chemistry, University of Tabriz, \\ Tabriz, Iran
}

Received: 22 June 2021

Accepted: 28 August 2021

Published online:

7 September 2021

(C) The Author(s), under exclusive licence to Springer Science+Business Media, LLC, part of Springer Nature 2021

\begin{abstract}
Nowadays, hydrogels have been attracted a lot of interest due to their immense potential in different fields such as biomedicine and biotechnology. Biodegradable and biocompatible $\mathrm{pH}$-sensitive chitosan-grafted polyacrylic acid (CTS-g-PAA) hydrogel was synthesized by grafting an acrylic acid monomer onto chitosan at the presence of methylene bisacrylamide as a cross-linking agent and ammonium persulphate as an initiator. FT-IR spectroscopy and scanning electron microscopy (SEM) were used to analyze the properties of the obtained hydrogel. The synthesized hydrogel is suitable for the delivery of many hydrophilic drugs or species. Using a multi-walled carbon nanotube modified-glassy carbon electrode (CNT-GCE), the loading and release conditions of Nile Blue (NB) as an electroactive compound were evaluated utilizing the differential pulse voltammetry (DPV) and cyclic voltammetry (CV). The effect of various parameters on the electrochemical signal of NB was investigated, and the optimal conditions for the efficient performance of hydrogel to delivery of NB were obtained. The electrocatalytic current values show linear dependence to NB concentration in the range of $0.098-0.971 \mu \mathrm{M}$ while the detection limit of this electrochemical platform was $12.3 \mathrm{nM}$. The unique proposed hydrogel with the electroactive NB has a broad range of possible applications in biosensors for signal enhancement and bioanalysis.
\end{abstract}

Address correspondence to E-mail: i-alipour@tabrizu.ac.ir 


\section{Introduction}

Hydrogels are cross-linked, three-dimensional polymer networks. Since they contain multiple hydrophilic groups, they have a high affinity for water, allowing them to absorb large quantities of water, up to more than $20 \%$ of their weight. They can also adsorb other bodily fluids [1]. Due to the formation of physical or chemical bonds between polymer chains, they are insoluble in water. These polymer networks are cross-linked via physical or chemical interactions. Chemically cross-linked hydrogels are typically made by photo, thermal, or initiated free-radical polymerization, while physically cross-linked networks are made through non-covalent interactions at the molecular level [2]. In addition, these polymer networks have a wide range of applications, ranging from daily life to advanced large industries [3]. Their physical properties such as permeation, swelling, surface specifications, and mechanical strength can be adjusted by structural modifications [4]. Based on the preparation source, hydrogels are divided into two natural and synthetic categories [5]. Hydrogels based on natural polymers have recently attracted a lot of interest due to their controlled delivery of bioactive molecules, biocompatibility, and tissue engineering capabilities [6]. Chitosan is a natural linear copolymer of glucosamine and $\mathrm{N}$-acetyl glucosamine obtained by deacetylation of chitin, which is the second most abundant polysaccharide in nature after cellulose [7]. It is a weak base that has a solubility in an acidic $(\mathrm{pH}<6.5)$ medium and is insoluble in water and other organic solvents [8]. Because the synthesized hydrogel has several intrinsic properties such as sensitivity to $\mathrm{pH}$, biocompatibility, low toxicity, biodegradability and, bioadhesion, so it can be applied in various domains such as biomedical, biological, pharmaceutical, and analytical fields [9]. Modified chitosan hydrogels are excellent carriers for delivering a variety of drug molecules [10]. Many studies have been published on chitosan hydrogel modifications and their applications in the delivery of various compounds, as described in ref [11-16]. One of the main challenges in biomedical sciences in the diagnosis of severe diseases such as cancers is the existence of their relevant biomarkers at extremely low concentrations in the early stages of the disease, which are below the detection limits of many analytical techniques. Signal enhancement using drug carriers such as liposomes, hydrogels, polymersomes etc. $[17,18]$ is one of the most effective ways to improve these techniques' detection limits. These carriers can be loaded with a variety of marker molecules, and they are connected to the detection system using a suitable detection strategy. The related analytical signal of the released indicators is then recorded by providing optimal conditions for the release of the maximum number of indicator molecules. The obtained signal is proportional to the biomarker concentration in these biosensors. Instead of one or more indicator molecules, millions of indicator molecules are released from a single drug carrier which is recorded for each biomarker molecule. As a result, identification of such biomarkers at very low concentrations may be possible, increasing the chances of early diagnosis of diseases.

Some studies have reported the use of carriers such as liposomes in electrochemical biosensors that is loaded with electroactive markers. Esmaeel Alipour et al. used dopamine-loaded liposome as a signal enhancer in the detection of oligonucleotides [19]. Mohammadreza Alizade-ghodsi and co-workers presented an electrochemical method based on signal amplification using liposome as a carrier for the detection of human telomerase activity [20]. More information about these carriers for electrochemical signal enhancement and also their applications in biomarkers detection are in ref [21-24]. Since the carrier systems may load large amounts of electroactive indicators, it appears that by releasing a large amount of them (via physical or chemical changes), the electrochemical signal obtained can be amplified by several orders of magnitude. This strategy is critical for the sensitivity of electrochemical biosensors that are used to determine the key biological species. This study aimed to see if the $\mathrm{pH}$ sensitive CTS-g-PAA hydrogel could be used to load and release NB as an electroactive indicator by considering the prospect of being used as a carrier device for signal enhancement. The synthesized hydrogel was characterized and the differential pulse voltammetry studies were performed using the carbon nanotube modified glassy carbon electrode to assess the loading and release amounts of NB. In addition, the electrocatalytic activity of NB toward the modified electrode was studied in detail. 


\section{Materials and methods}

\subsection{Materials}

All the chemical materials including potassium dihydrogen phosphate $\left(\mathrm{KH}_{2} \mathrm{PO}_{4}\right)$, sodium hydrogen phosphate $\left(\mathrm{Na}_{2} \mathrm{HPO}_{4}\right)$, boric acid $\left(\mathrm{H}_{3} \mathrm{BO}_{3}\right)$, acetic acid $\left(\mathrm{CH}_{3} \mathrm{COOH}\right)$, Nile blue $\left(\mathrm{C}_{20} \mathrm{H}_{20} \mathrm{CIN}_{3} \mathrm{O}\right)$, methylene bisacrylamide $\left(\mathrm{C}_{7} \mathrm{H}_{10} \mathrm{~N}_{2} \mathrm{O}_{2}\right)$, ammonium persulphate $\left(\left(\mathrm{NH}_{4}\right)_{2} \mathrm{~S}_{2} \mathrm{O}_{8}\right)$, chitosan, $\mathrm{HCl}, \mathrm{NaOH}$ and ethanol used in the present work were prepared from Merck.

\subsection{Apparatus}

All electrochemical studies were performed using Autolab (model PGSTAT 30, Netherland) that was controlled by GPES software (4.9 version) to record voltammetric information. All three electrodes consist of a saturated calomel electrode as a reference, glassy carbon as working, and platinum as the counter electrodes were purchased from Azar electrode company (Urmia, Iran). A magnetic vortex (model 649, Metrohm, Switzerland) was used for stirring and homogenizing solutions/suspensions. For gel separation in hydrogel synthesis, centrifuge Hettich (model EBA) was applied. In addition, the $\mathrm{pH}$ meter (model 627, Metrohm) was used for adjusting the $\mathrm{pH}$ of solutions.

\subsection{Procedure}

\subsubsection{Preparation of carbon nanotubes}

Carbon by-products and catalytic residues are two main sources of carbon nanotubes (CNTs) impurities. Therefore, the acid treatment was used to remove impurities leftover from the synthesis process. To do this, $0.500 \mathrm{~g}$ of CNTs were added to the mixture containing $20 \mathrm{~mL} \mathrm{HNO}_{3}$ and $20 \mathrm{~mL} \mathrm{H}_{2} \mathrm{SO}_{4}$ (1:1), and the suspension was sonicated in two steps: first for $3 \mathrm{~h}$ at $55^{\circ} \mathrm{C}$ and then for $2 \mathrm{~h}$ at $80^{\circ} \mathrm{C}$ [25]. Furthermore, besides using filter paper, it was washed with distilled water until it was adjusted to the neutral $\mathrm{pH}$. Subsequently, DMF was utilized as a dispersing solvent for obtaining a homogenous and relatively uniform suspension of CNTs for further modification of the electrode surface. In this regard, the black powder was dried in an oven for one day, and $4 \mathrm{mg}$ of the nanotube powder and $2 \mathrm{~mL}$ DMF was stirred ultrasonically for $2 \mathrm{~h}$. Finally, modification of electrode was performed by applying $4 \mu \mathrm{L}$ of prepared CNTs on its surface followed by rinsing with distilled water for $20 \mathrm{~min}$. Schematic illustration of the CNTs preparation is presented in Fig. S1A.

\subsubsection{Synthesis of NB-loaded CTS-g-PAA hydrogel}

$1.0 \mathrm{~g}$ of chitosan was slowly applied to the stirring $25 \mathrm{~mL}$ acetic acid solution $(1 \% \mathrm{v} / \mathrm{v})$ of $70{ }^{\circ} \mathrm{C}$ and kept for $15 \mathrm{~min}$ to dissolve completely. Following that, $0.625 \mathrm{~g}$ of NB (1.954 mmoles) was added and stirred for 20 min. $0.0184 \mathrm{~g}$ methylene bisacrylamide (MBA) as a cross-linking agent and $1.84 \mathrm{~mL}$ of acrylic acid (AA) as a monomer was added to the above-mentioned mixture solution simultaneously and stirred for $15 \mathrm{~min}$. Ammonium persulfate (APS, $0.054 \mathrm{mg}$ ) was used to initiate the polymerization process. The reaction was allowed to proceed for $2 \mathrm{~h}$ at room temperature. After that, the mixture was neutralized with $1.7 \mathrm{M} \mathrm{NaOH}$. The obtained product was held in $120 \mathrm{~mL}$ ethanol for $24 \mathrm{~h}$ to eliminate water and other impurities, after which it was filtered and dried fully at $60{ }^{\circ} \mathrm{C}$ for $24 \mathrm{~h}$. The filtrate was diluted 100 times with distilled water (d-filtrate) and used for voltammetric determination of unloaded NB, following by quantification of amounts of loaded NB in the hydrogel. Schematic illustration of NB-loaded CTS-gPAA hydrogel synthesis is presented as Fig. S1B.

\subsubsection{Measuring the loaded NB}

Proper volume (e.g., $20 \mu \mathrm{L}$ ) of the d-filtrate solution was added to an electrochemical cell containing $3 \mathrm{~mL}$ of background electrolyte including phosphate buffer $(\mathrm{pH}=6)$ with an overall concentration of $0.1 \mathrm{M}$ and $0.025 \mathrm{M} \mathrm{NaCl}$. Then, using the DPV technique and the standard addition method, the concentration of unloaded NB was determined. Finally, the unloaded NB mole content in the filtrate solution was calculated. (unloaded NB moles).

\subsubsection{Measuring the released NB}

To release NB that had been loaded in the hydrogel during the synthesis process, $10 \mathrm{~mL}$ britton-robinson buffer with various $\mathrm{pHs}$ was added to $0.01 \mathrm{gr}$ of synthesized hydrogel, which was then filtered for separation of the supernatant solution after $24 \mathrm{~h}$ (NB released solution). The concentration of released NB was then determined using differential pulse 
Fig. 1 DPVs of $1 \mu \mathrm{M} N B$ recorded using: a bare GCE, and $\mathbf{b}$ CNT-GCE. Background electrolyte: $0.1 \mathrm{M}$ brittonrobinson buffer $\mathrm{pH}=7$ containing $0.2 \mathrm{M} \mathrm{NaCl}$

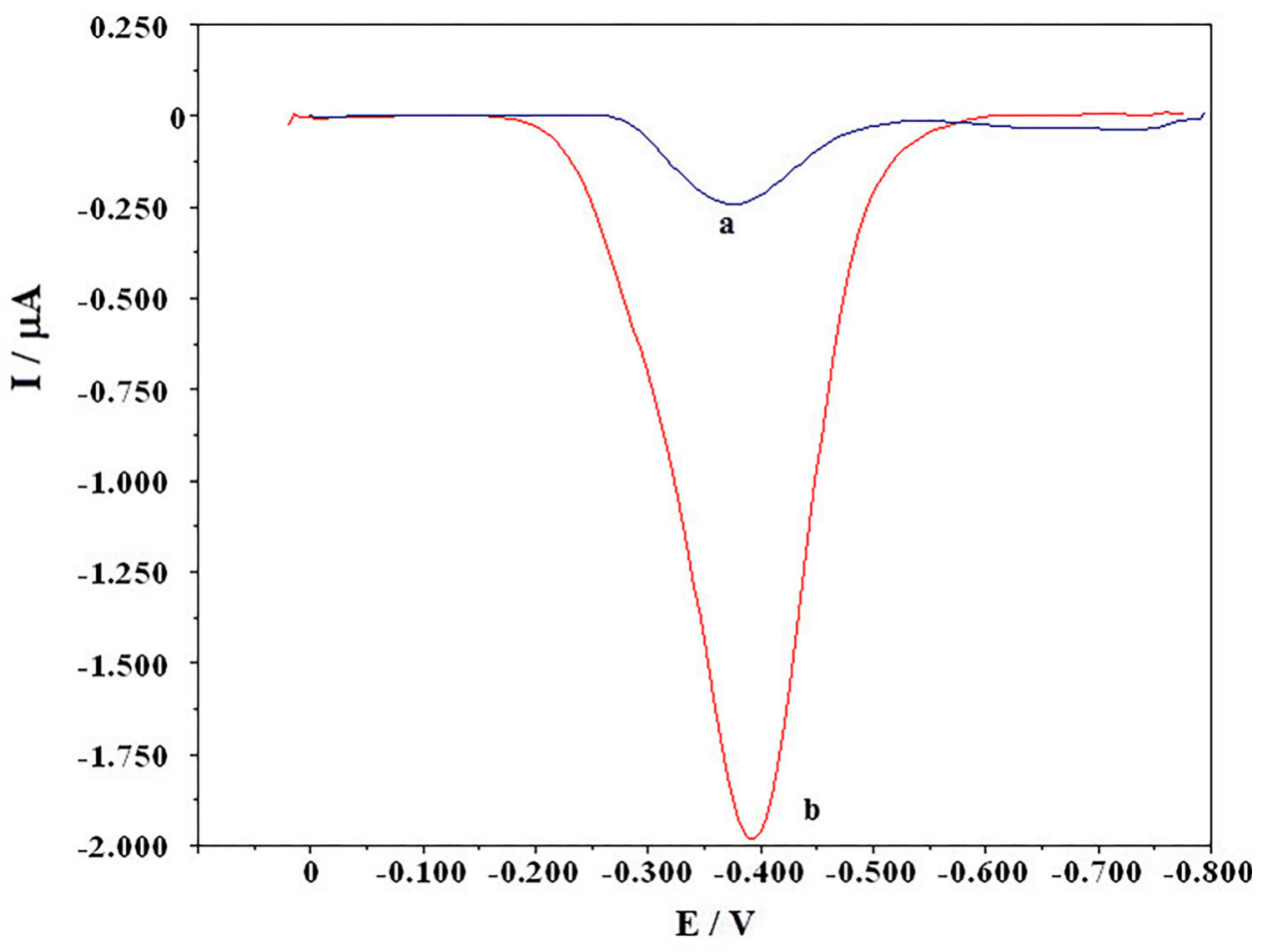

voltammetry (DPV) and the standard addition procedure.

\subsubsection{Electrochemical studies}

\subsubsection{Differential pulse voltammetry First CNT-GCE} was immersed in a stirring solution of $0.1 \mathrm{M}$ phosphate buffer $(\mathrm{pH}=7)$ containing $0.025 \mathrm{M} \mathrm{NaCl}$ and $1.5 \mu \mathrm{M}$ NB for 4 min and then its DP voltammogram was recorded at the potential ranging from $(0.0-0.8 \mathrm{~V})$ with scan rates of $25 \mathrm{mV} / \mathrm{s}$. After that, the so-called NB-CNT-GCE was used to measure different NB concentrations. It should be noted that the calibration curves were drawn using normalized currents; the CNT-GCE was immersed in a stirring solution of $1.5 \mu \mathrm{M} \mathrm{NB}$ for $4 \mathrm{~min}$ and after recording its DP voltammogram, the subsequent peak current $\left(i_{b}\right)$ was determined. Then, multiplex concentrations of NB were applied and the associated peak currents $\left(i_{p}\right)$ were measured. Finally, the calibration curve of $\Delta \mathrm{i}=\mathrm{i}_{\mathrm{p}}-\mathrm{i}_{\mathrm{b}}$ was plotted versus the NB concentration.

\subsubsection{ChronoAmperometry (ChA) The modified} electrode was put in an electrochemical cell of blank buffer solution containing $0.25 \mathrm{mM} \mathrm{NB}$ and the following chronoamperometric measurement was performed at the potential of $-0.62 \mathrm{~V} / \mathrm{SCE}$ for $100 \mathrm{~s}$.

\section{Results and discussion}

\subsection{Preliminary investigation}

DPVs of $1 \mathrm{M} \mathrm{NB}$ were recorded using bare GCE (Fig. 1, curve a) and CNT-GCE (Fig. 1, curve b) to study the electrocatalytic activity of CNT- GCE against NB. It can be observed that while using the CNT-GCE, the electrochemical activity of NB is more desirable and the modified electrode shows higher electrochemical performance. Thus, the CNT-GCE was used as the working electrode in the following experiments.

\subsection{Effect of experimental conditions on the peak current of NB}

\subsubsection{Effect of NB concentration}

Different concentrations of NB were prepared and their DPVs (Fig. 2), as well as their corresponding calibration curves, were obtained, to investigate the effect of NB concentration (Fig. 2, inset A). As it can be seen in Fig. 2, A, there are two linear sections. The first part has a lower slope than the second part. It seems that NB first adsorbed on the modified CNTGCE surface, so the reduction of other NB molecules was facilitated on the NB-CNT-GCE surface. Thus, 
Fig. 2 DPVs of CNT-GCE in the presence of various concentrations of NB (a to $\mathrm{k}$ ): $0.33,0.66,0.99,1.32,1.64$, $1.96,2.28,2.60,2.91,3.22$ and $3.53 \mu \mathrm{M}$. Background electrolytes cited in Fig. 1. The time between two consecutive NB injections: 4 min. Inset $\mathbf{A}$ related calibration curve. Inset B variation of $\Delta \mathrm{I}$ versus NB concentration

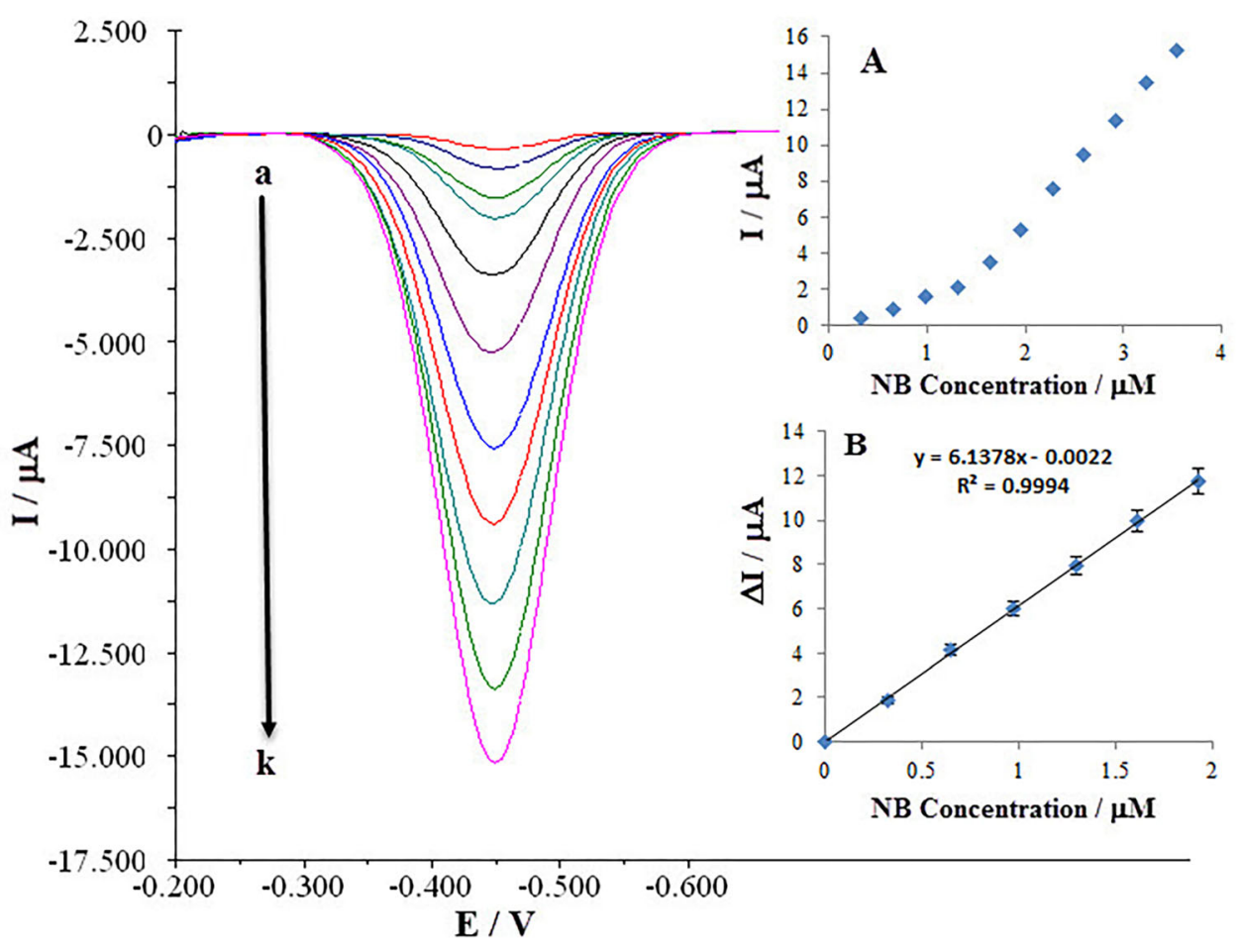

the NB-CNT-GCE was used to measure various NB concentrations. It should be remembered that the normalized currents were used for drawing the calibration curves; the CNT-GCE was immersed in a stirring solution of $1.5 \mu \mathrm{M} \mathrm{NB}$ for $4 \mathrm{~min}$ and its DP voltammogram and subsequent peak current $\left(i_{b}\right)$ was determined. Then multiplex concentrations of NB were applied and peak currents $\left(i_{p}\right)$ associated with them were measured. Finally the calibration curve of $\Delta \mathrm{i}=\mathrm{i}_{\mathrm{p}}-\mathrm{i}_{\mathrm{b}}$ was plotted versus the NB concentration (Fig. 2, inset B). It is clear that employing NB-CNTGCE, the obtained results are more favorable.

\subsubsection{Effect of supporting electrolyte concentration}

The presence of a supporting electrolyte is needed in voltammetric measurements to reduce solution resistance, eliminate the effects of analyte migration, and obtain a constant ionic force. $\mathrm{NaCl}$ was used as a supporting electrolyte in this study. The influence of the $\mathrm{NaCl}$ concentration on the reduction peak currents of NB was investigated using the brittonrobinson buffer $0.1 \mathrm{M}(\mathrm{pH}=7)$. Thus at a given concentration of supporting electrolyte and increasing amounts of NB, DPVs were recorded using NBCNT-GCE and their calibration curves were obtained. The magnitude of the calibration curve slopes was used to determine the best supporting electrolyte concentration. Table 1 lists the DPV results obtained for various $\mathrm{NaCl}$ concentrations, as well as the calibration curves for each. According to the results, it is clear that $0.025 \mathrm{M} \mathrm{NaCl}$ has the best analytical sensitivity. Thus, $0.025 \mathrm{M}$ was chosen as the optimal $\mathrm{NaCl}$ concentration.

\subsubsection{Effect of $p H$}

Different pHs of the Britton-Robinson buffer were checked to see how $\mathrm{pH}$ affected the peak current of NB. Using DPV technique, various calibration curves for NB measurements were obtained at different $\mathrm{pHs}$. The histogram of corresponding calibration curve slopes versus $\mathrm{pH}$ is shown in Figure S2A. The results indicate that the maximum sensitivity (slope) is gained at $\mathrm{pH}=7$ while its minimum value is at $\mathrm{pH}=12$. Figure S2B also shows a diagram of the NB reduction potential at different $\mathrm{pHs}$. Since the slope of this diagram (0.0601) is nearly identical to Nernst's slope, it is assumed that the number of electrons and protons shared in NB is the same.

Following that, the effect of buffer type on the NB peak current was investigated. In the presence of various NB concentrations, the DPVs of the NB were obtained using three different $0.1 \mathrm{M}$ buffers (brittonrobinson, phosphate, and Tris) with $\mathrm{pH}=7$. The slope of the calibration curve while using the phosphate buffer was noticeably higher than that of the others (it is approximately more than twofold the 
Table 1 Effect of $\mathrm{NaCl}$ concentration on the sensitivity of the sensor

\begin{tabular}{lcc}
\hline $\mathrm{NaCl}$ concentration $(\mathrm{M})$ & Calibration curve slope $(\mu \mathrm{A} / \mu \mathrm{M})$ & $\mathrm{R}^{2}$ \\
\hline 0 & 9.126 & 0.9975 \\
0.0125 & 10.515 & 0.9947 \\
0.025 & 20.715 & 0.9995 \\
0.062 & 13.461 & 0.9981 \\
0.125 & 11.201 & 0.9966 \\
0.2 & 6.138 & 0.9994 \\
\hline
\end{tabular}

slope related to the britton-robinson buffer). As a consequence of the findings, phosphate buffer was used in subsequent experiments.

\subsubsection{Effect of scan rate}

The impact of potential scan rate on NB reduction current was studied. Figure 3A shows cyclic voltammograms of CNT-GCE in a $1 \mathrm{mM}$ NB solution containing $0.1 \mathrm{M}$ phosphate buffer $(\mathrm{pH}=7)$ and $0.025 \mathrm{M} \mathrm{NaCl}$, with scan rates ranging from 10 to $200 \mathrm{mV} / \mathrm{s}$. Both Anodic and cathodic peak currents of NB are increased simultaneously by scan rates. The kinetic polarization of the electron transfer mechanism is indicated by an increase in anodic peak potential and a decrease in cathodic peak potential at higher scan rates. The diagram of cathodic peak currents of NB versus scan rates (inset $\mathrm{x}$ ) and also versus the square root of scan rates (inset y) using the CNT-GCE is shown in the insets of Fig. 3A. The cathodic peak current variations are almost linear against both scan rate and root square of scan rate at the studied potential scan rates. Further studies have also confirmed that at lower scan rates the cathodic peak currents show a better linear correlation with scan rate. It means that at lower scan rates, adsorption controls the current. On the other hand, the peak current variations have better linearity versus the root square of scan rate at higher scan rates (Fig. 3B), implying that the current is diffusive at higher scan rates. Figure 3C demonstrates the plot of log I versus $\log v$ at the scan rates ranging from $10-200 \mathrm{mV} / \mathrm{s}$. It should also be noted that the slope of the described plot is approximately 1 for adsorptive currents and about 0.5 for diffusive currents. So, according to the slope of the plot in Fig. 3C, the electrode reactions are under the influence of both adsorption and diffusion at the studied scan rates. However, the adsorption one makes a larger contribution to the overall current.
The charge transfer coefficient $(\alpha)$ was calculated as a kinetic parameter in the next step. CNT-GCE was immersed in a solution containing $0.1 \mathrm{M}$ phosphate buffer $(\mathrm{pH}=7), 0.025 \mathrm{M} \mathrm{NaCl}$, and $1 \mathrm{mM} \mathrm{NB}$, and cyclic voltammetry was conducted at a low scan rate of $30 \mathrm{mV} / \mathrm{s}$. A straight line was obtained using the Toffel curve (Fig. 3D), which is a graph of log I versus $\mathrm{E}$ (using the ascendant portion of CV). Based on this line slope, and assuming $n=1$, the value of $\alpha=0.24$ was calculated in the rate-determining step.

\subsection{Calculation of the effective surface area of the CNT-GCE}

The Randles-Sevcik equation (Eq. 1) was utilized to quantitatively determine the effective surface area of the electrode [26]. This equation describes the linear relationship between peak current $\left(I_{p}\right)$ and the square root of the scan rate $\left(\vartheta^{1 / 2}\right)$. In this regard, cyclic voltammetry of CNT-GCE was done using $10 \mathrm{mM}$ potassium hexacyanoferrate and $0.2 \mathrm{M} \mathrm{NaCl}$. Using the obtained cyclic voltammograms and the slope of the graph of I versus square root of $\vartheta$ (Figure S3) and also according to Randles-Sevcik equation, the value of $\mathrm{A}=0.2819 \mathrm{~cm}^{2}$ was estimated. Given that the geometrical surface area of the GCE is $0.0314 \mathrm{~cm}^{3}$, it seems that modifying with CNTs increases the effective surface area of the electrode by 9 times resulting in a large number of active sites for NB reduction.

$I_{p}=2.65 \times 10^{5} n^{3 / 2} A C D^{1 / 2} \vartheta^{1 / 2}$

\subsection{Chronoamperometric studies}

The diffusion coefficient of NB was determined utilizing chronoamperometry. By applying a negative potential step of $-0.62 \mathrm{~V}$, a chronoamperogram of $0.5 \mathrm{mM} \mathrm{NB}$ was recorded. Using this chronoamperogram, the graph of $\mathrm{I}$ versus $\mathrm{t}^{-1 / 2}$ was obtained (Figure S4). From this figure and according to the 

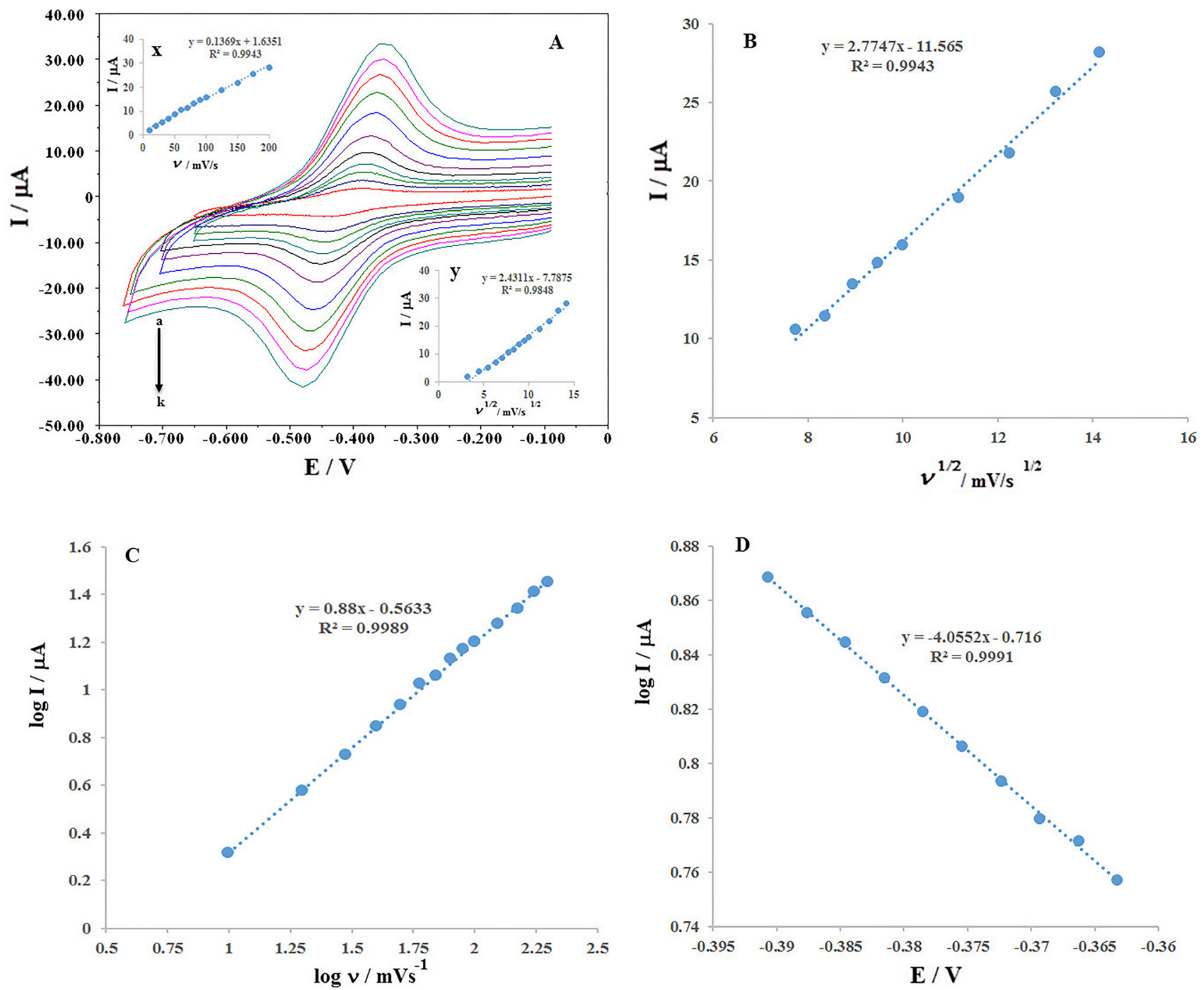

Fig. 3 A Cyclic voltammetric responses of CNT-GCE in $0.1 \mathrm{M}$ phosphate buffer $\mathrm{pH}=7$ containing $0.025 \mathrm{M} \mathrm{NaCl}$ in the presence of $1 \mathrm{mM} \mathrm{NB}$ at scan rates of (a-k): 10, 20, 30, 40, 50, 70, 100, $125,150,175,200 \mathrm{mV} \mathrm{s}^{-1}$. Inset $\mathrm{x}$ : The plot of cathodic peak current vs. scan rate. Inset y: The plot of cathodic peak current vs.

Cottrell equation (Eq. 2) [27], the value of NB diffusion coefficient, $\mathrm{D}=4.06 \times 10^{-6} \mathrm{~cm}^{2} / \mathrm{s}$ was calculated.

$I=\frac{n F A C D^{1 / 2}}{\pi^{1 / 2} t^{1 / 2}}$

\subsection{Analytical performances}

To evaluate the analytical performance of the developed electrode, the addition of various NB concentrations was carried out. Figure 4, presents the DPV $v^{1 / 2}$. B The cathodic peak current vs. $v^{1 / 2}$ at scan rates of 50 $200 \mathrm{mV} \mathrm{s}^{-1}$. C Variation of cathodic peak current logarithm versus scan rate logarithm. D Tafel plot derived from the rising part of the voltammogram c shown in (A)

measurements of different concentrations of NB ranging from $0.098 \mu \mathrm{M}$ to $0.971 \mu \mathrm{M}$ using the NBCNT-GCE. Also, the respective calibration curve is shown as an inset in Fig. 4. It should be noted that the calibration curves were drawn using normalized currents; the CNT-GCE was immersed in a stirring solution of $1.5 \mu \mathrm{M}$ NB for $4 \mathrm{~min}$ and its DP voltammogram and subsequent peak current $\left(i_{b}\right)$ was determined. After that, different concentrations of NB were applied, and the corresponding peak currents $\left(i_{p}\right)$ were recorded. Finally the calibration curve of $\Delta \mathrm{i}=\mathrm{i}_{\mathrm{p}}-\mathrm{i}_{\mathrm{b}}$ was plotted versus the NB concentration 
Fig. 4 DPVs of NB-CNTGCE in $0.1 \mathrm{M}$ phosphate buffer $\mathrm{pH}=7$ containing $0.025 \mathrm{M} \mathrm{NaCl}$ and in the presence of various concentrations of NB (a-k): 0 , 0.098, 0.196, 0.293, 0.391, $0.488,0.585,0.682,0.778$, 0.875 and $0.971 \mu \mathrm{M}$. Time between two consecutive NB injections: $4 \mathrm{~min}$. Inset: related calibration curve

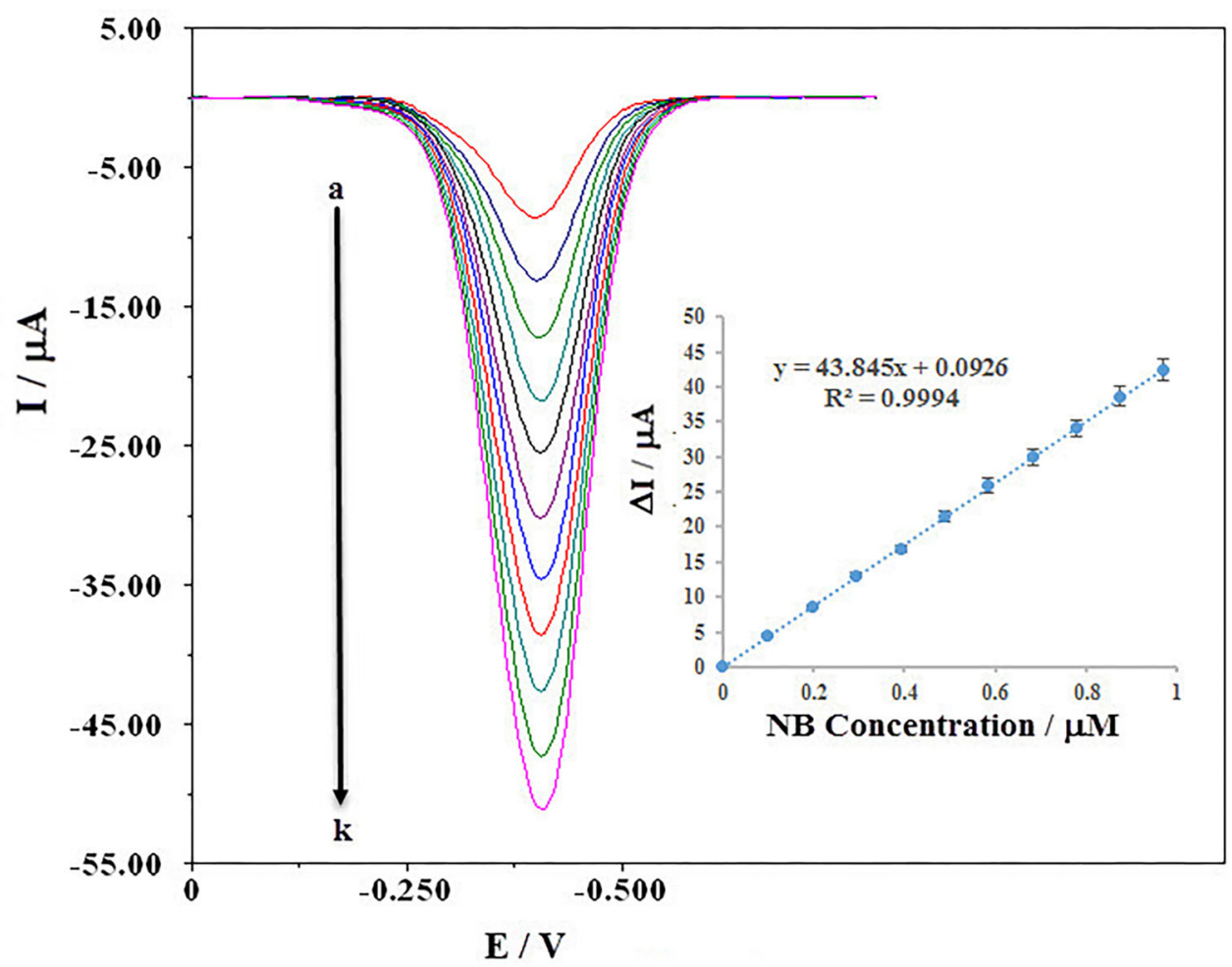

(inset of Fig. 4). The obtained results show that the linear range is between $(0.098-0.971) \mu \mathrm{M}$ of NB. In addition, Eq. 3 was applied to calculate the LOD of the method [28].

$L O D=\frac{3 S_{y / x}}{b}$

in which $S_{y / x}$ is corresponding to the standard deviation of the response and $b$ is the slope of the calibration curve. Therefore, using Eq. 3, the detection limit for NB is found to be $12.3 \mathrm{nM}$.

\subsection{Investigation of the required conditions for utilization of NB as an indicator in biosensor preparation}

Higher sensitivities and lower detection limits are critical in analytical chemistry and, in particular, in biosensor design. Signal amplification is one of the effective strategies for achieving this aim. Recently carrier systems have attracted much attention for their applications as signal amplifiers [19, 29, 30]. They can encapsulate various types of markers like enzymes, dyes, electrochemical or chemiluminescent compounds, and so on. The ability to load millions of marker molecules into a single carrier molecule causes the analytical signal to increase for many orders of magnitude, resulting in the detection of lower concentrations of intended analytes (analyte concentration is proportional to analytical signal). As a result, determining the conditions under which the greatest number of marker molecules can be loaded onto these carriers and then released, is becoming increasingly important.

\subsubsection{Physicochemical characterization of CTS-g-PAA hydrogel}

Figure S5 (A and B) show the FT-IR spectra of the chitosan and synthesized CTS-g-PAA hydrogel, respectively. In contrast with the chitosan (Fig. S5A), several new peaks were observed from the FT-IR spectrum of CTS-g-PAA hydrogel (Fig S5B). Two peaks at $1546 \mathrm{~cm}^{-1}$ and $1401.2 \mathrm{~cm}^{-1}$ can be assigned to the asymmetric and symmetric stretching vibration of $-\mathrm{COO}^{-}$groups, respectively. In addition, a peak of $1691 \mathrm{~cm}^{-1}$ can be specified to the symmetric vibration of the $\mathrm{C}=\mathrm{O}$ group of hydrogel structure. A peak at $1740 \mathrm{~cm}^{-1}$ is attributed to the amide group, because of cross-linking effect. A wide absorption peak at $3429 \mathrm{~cm}^{-1}$ is due to the vibration of the $-\mathrm{OH}$ group. In addition, a low-intensity absorption peak at $2880-2921 \mathrm{~cm}^{-1}$ corresponds to the C-H 
Fig. 5 DPVs of: (a) NB-CNTGCE, and (a) after addition of $20 \mu \mathrm{l}$ of the d-filtrate solution in $3 \mathrm{ml}$ of background electrolyte $(0.1 \mathrm{M}$ phosphate buffer $\mathrm{pH}=7$ containing $0.025 \mathrm{M} \mathrm{NaCl}$ ), and after standard addition of various concentrations of NB (c to g): $0.32,0.65,0.97,1.29$ and $1.60 \mu \mathrm{M}$. The time between two consecutive NB injections: $4 \mathrm{~min}$. Inset: related calibration curve

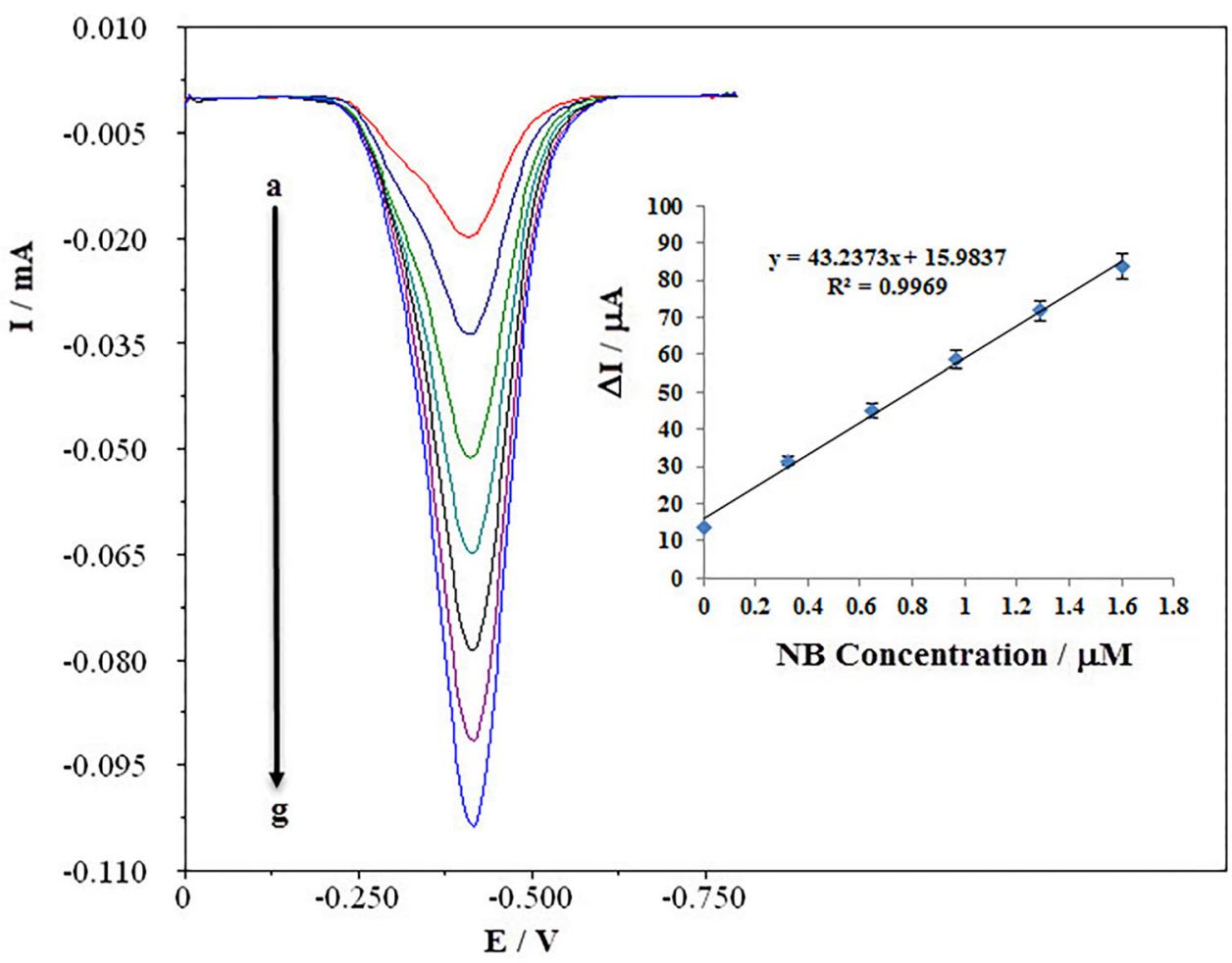

group [31, 32]. A comparison of the two FTIR spectra confirms the formation of the proposed hydrogel.

The surface morphology of the resultant hydrogel was performed using scanning electron microscopy (SEM). Figure S6 shows the SEM image of the synthesized hydrogel before (Figure S6A) and after loading of NB (Figure S6B). The SEM results showed the wrinkled sheet morphology of the synthesized hydrogel. The existence of cavities on the hydrogel surface and in the internal zones exhibited a porous structure. Cavities cause a larger adsorption capacity at hydrogel which can be seen in the synthesized CTS-g-PAA hydrogel.

After the loading process, significant changes in the hydrogel surface were monitored, which could be due to the adsorption of NB. Therefore, it can be stated that the manufactured hydrogel has a suitable ability to adsorb NB.

\subsubsection{NB loading studies}

Because of the hydrophilic nature of NB, it was successfully loaded into the hydrogel network and the amount of loading played a key role in achieving higher current signals in subsequent voltammetric analysis. For determination of the loaded NB amount, $20 \mu \mathrm{L}$ of the d-filtrate solution (see Sect. 2.2.2) was applied into an electrochemical cell containing $3 \mathrm{~mL}$ of background electrolyte including $0.1 \mathrm{M}$ phosphate buffer $(\mathrm{pH}=7)$ and $0.025 \mathrm{M} \mathrm{NaCl}$. Then, using the DPV technique and the standard addition method, the concentration of the unloaded NB was calculated (Fig. 5). The inset of Fig. 5 corresponds to the related standard addition graph. According to this analysis, the obtained NB concentration within the cell was $0.37 \mu \mathrm{M}$. Thus, the amount of NB into the $\mathrm{d}$-filtrate solution was estimated to be $55.87 \mu \mathrm{M}$ that is equivalent to the $5.587 \mathrm{mM}$ in the $120 \mathrm{~mL}$ of filtrate ethanol (equivalent to $670.44 \mu$ mole which is not loaded, see Sect. 2.2.2). As a result, from the total sum of 1.954 mmoles used during the hydrogel synthesis, 1.283 mmoles of NB were successfully loaded into the hydrogel structure $(\%$ Loading $=65.7)$. In other words, $1.250 \mathrm{~g}$ of dried hydrogel contains $1.283 \mathrm{~mol}$ NB which means that each $1 \mathrm{~g}$ of dried hydrogel contains 1.022 mol NB.

\subsubsection{NB release studies}

As mentioned above, to improve the detection limits of DNA biosensors, signal enhancement strategies are critical. Using carrier systems such as liposomes, polymersomes, hydrogels, and others is one of the most effective approaches. A number of studies have 
Table 2 Specifications of recent studies used carrier systems as signal amplification agent

\begin{tabular}{|c|c|c|c|c|}
\hline Carrier & $\begin{array}{l}\text { Loaded } \\
\text { material }\end{array}$ & Detection strategy & Detected biomarker & References \\
\hline Liposome & Dopamine & Electrochemical & Oligonucleotide & [19] \\
\hline Liposome & Dopamine & Electrochemical & Telomerase & {$[20]$} \\
\hline Liposome & Quantum dot & Optical & Telomerase & {$[23]$} \\
\hline Liposome & Calcium ions & Electrochemical & E. Coli bacteria & {$[24]$} \\
\hline Liposome & HRP enzyme & Electrochemical & Cancer antigen & {$[33]$} \\
\hline Liposome & $\mathrm{V}_{2} \mathrm{O}_{5} \mathrm{NPs}$ & $\begin{array}{l}\text { Dual colorimetric- } \\
\text { electrochemical }\end{array}$ & Virus & {$[34]$} \\
\hline Polymersome & Pd-Ir NPs & Colorimetric & Human prostate antigen & {$[35]$} \\
\hline Gold vesicles & Fuchsine dye & Colorimetric & Covid -19 & {$[36]$} \\
\hline Hydrogel & Nile blue & electrochemical & $\begin{array}{l}\text { Can be used in DNA hybridization biosensors and } \\
\text { aptasensors }\end{array}$ & This study \\
\hline
\end{tabular}

been conducted employing various carrier systems as signal amplification agents are given in Table 2. For example, the electroactive indicator loaded carrier system was combined with the DNA reporter probe in the development of the electrochemical DNA hybridization biosensors.

Assuming that one carrier per target was attached to the detection system, and also assuming that each carrier is encapsulated millions of electroactive indicator molecules, so as a result, millions of indicator molecules per target can be released. Thus a very large signal can be obtained per a target. As a consequence, very low detection limits can be obtained enabling the early detection of severe diseases biomarkers. In this regard, to demonstrate the capability of the proposed CTS-g-PAA hydrogel as a carrier system of NB, understanding the NB loading and release behavior under various conditions is necessary.

Therefore, the impact of $\mathrm{pH}$ and time on NB release was investigated in this section of the report. Since the synthesized hydrogel is sensitive to $\mathrm{pH}, \mathrm{NB}$ release was tested using the britton-robinson buffer at $\mathrm{pHs}$ ranging from 1 to 12 . To determine the amount of NB released, $10 \mu \mathrm{L}$ of the NB release solution (see Sect. 2.2.3) was added to an electrochemical cell containing $3 \mathrm{~mL}$ of background electrolyte $(0.1 \mathrm{M}$ phosphate buffer $(\mathrm{pH}=7)$ and $0.025 \mathrm{M}$ $\mathrm{NaCl}$ ). Then, using the DPV technique and the standard addition method, the concentration of NB was calculated. The histogram of NB release percent at various pHs is shown in Fig. 6A.

The amount of NB release is significantly higher at $\mathrm{pH}=6$. Since the swelling of hydrogels is dependent on the electrostatic repulsion of charged species in
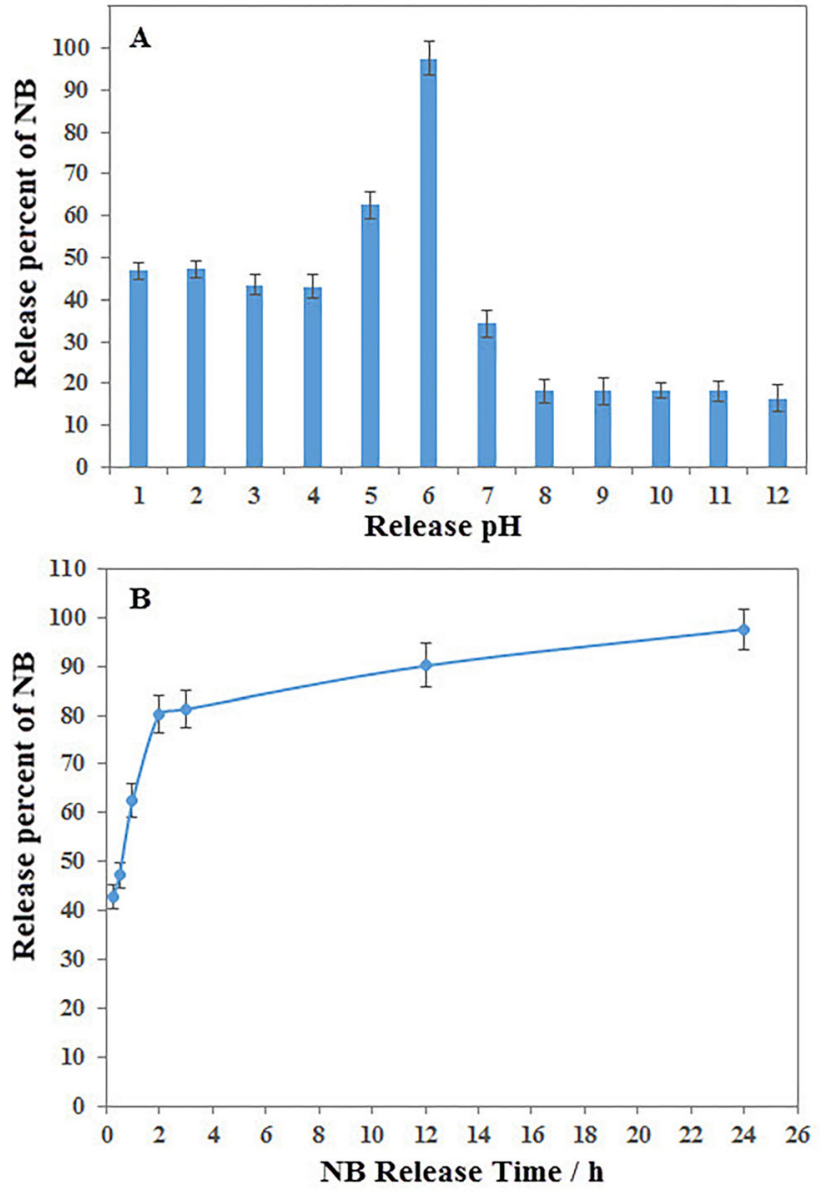

Fig. 6 A Histogram of release percent of NB at different release pHs. B Variation of release percent of NB versus NB release time

hydrogel structure, any condition that reduces this repulsion, such as $\mathrm{pH}$, counter ions, and ionic pressure, affects the degree of swelling. The significant changes in swelling are due to the existence of a variety of $\mathrm{pH}$-dependent interactions in its 
environment. Since the amine groups of chitosan (C2) protonated at low $\mathrm{pH}$, the chain-chain electrostatic repulsion (between $\mathrm{NH}_{3}{ }^{+}-\mathrm{NH}_{3}{ }^{+}$) increased, increasing the osmotic pressure within the hydrogel. $\mathrm{Cl}^{-}$ ions neutralize ammonium ions at very low $\mathrm{pH}$ $(\mathrm{pH}=4)$ and cause the effective repulsion to decrease. Similarly, at $\mathrm{pH}=6$, the ionization of carboxyl groups $\left(-\mathrm{COO}^{-}\right)$increases the hydrogel swelling by establishing the electrostatic repulsion. The presence of counter ions $\left(\mathrm{Na}^{+}\right)$at $\mathrm{pH}=8-12$ restricted the swelling capacity. As a result, large ionic concentrations at low and high $\mathrm{pH}$ resulted in an increased ionic strength, which was accompanied by a reduction in the osmotic pressure difference between the hydrogel and its surroundings, causing the swelling to decrease.

Also to investigate the impact of time on $\mathrm{NB}$ release, $10 \mathrm{~mL}$ britton-robinson buffer $(\mathrm{pH}=6)$ was applied to the $0.01 \mathrm{gr}$ of dried NB loaded hydrogel, which was then filtered after a certain period and the supernatant solution (NB released solution) separated. Then, $10 \mu \mathrm{L}$ of the NB released solution was added to an electrochemical cell containing $3 \mathrm{~mL}$ of background electrolyte. The level of NB was determined using the DPV technique and standard addition method. As the buffer diffused within the hydrogel and consequent swelling of the hydrogel, the loaded NB started to release steadily, which increases over time.

The results are shown in Fig. 6B. It is clear that as time passes, more NB is released from the hydrogel. Since there was no noticeable increase in the amount of released NB after $2 \mathrm{~h}$, this time ( $2 \mathrm{~h}$ ) was chosen as the optimum release time. As can be seen in Fig. 6B, the NB was released more than $40 \%$ after $10 \mathrm{~min}$ and more than $80 \%$ after $2 \mathrm{~h}$. It was estimated that $97.6 \%$ of NB was released during $24 \mathrm{~h}$. As previously stated, because the proposed system will be used in DNA hybridization biosensors that are currently being developed in our laboratory, shorter releasing times would reduce the overall analysis time. Higher release times will be used to determine targets with ultra-trace levels, while lower releasing times would suffice for detecting targets with moderate concentrations.

\section{Conclusion}

In this study, a new type of hydrogel based on chitosan and polyacrylamide was synthesized and it was used successfully for loading and release of the electroactive NB molecules. Highly sensitive DPV measurements along with CNT-GCE were used for electrochemical detection of released NB from the hydrogel. In addition, by considering the physicochemical properties of the synthesized hydrogel, it seems that the mentioned NB-loaded hydrogel can be applied to amplify the electrochemical signal of NB. The high loading yield of nearly millions of signaling NB molecules per carrier leads to sub-fM detection limits. Therefore, the proposed approach may provide a promising strategy for indirect and early detection of different biological biomarkers and also it can be used for the design of sensitive biosensors for early detection of fatal diseases. The utilizing of NB-loaded hydrogel for early detection of some cancers is in progress in our laboratory.

Supplementary Information: The online version contains supplementary material available at http s://doi.org/10.1007/s10854-021-06939-7.

\section{References}

1. E. Caló, V.V. Khutoryanskiy, Biomedical applications of hydrogels: A review of patents and commercial products. Eur. Polym. J. 65, 252-267 (2015)

2. S. Ma, B. Yu, X. Pei, F. Zhou, Structural hydrogels. Polymer (Guildf). 98, 516-535 (2016)

3. W.A. Laftah, S. Hashim, A.N. Ibrahim, Polymer hydrogels: A review. Polym. - Plast. Technol. Eng. 50, 1475-1486 (2011)

4. T.K. Giri, A. Thakur, A. Alexander et al., Modified chitosan hydrogels as drug delivery and tissue engineering systems: Present status and applications. Acta Pharm. Sin. B 2, 439-449 (2012)

5. A.K. Mahanta, P. Maiti, Injectable hydrogel through hydrophobic grafting on chitosan for controlled drug delivery. ACS Appl Bio Mater 2, 5415-5426 (2019). https://doi.org/10. 1021/acsabm.9b00733

6. N.M. Ranjha, G. Ayub, S. Naseem, M.T. Ansari, Preparation and characterization of hybrid $\mathrm{pH}$-sensitive hydrogels of chitosan-co-acrylic acid for controlled release of verapamil. J Mater Sci Mater Med 21, 2805-2816 (2010). https://doi.org/ 10.1007/s10856-010-4134-1 
7. M. Rinaudo, Chitin and chitosan: Properties and applications. Prog. Polym. Sci. 31, 603-632 (2006)

8. A.B. Nazlı, Y.S. Açıkel, Loading of cancer drug resveratrol to $\mathrm{pH}-$ Sensitive, smart, alginate-chitosan hydrogels and investigation of controlled release kinetics. J Drug Deliv Sci Technol 53, 101199 (2019). https://doi.org/10.1016/j.jddst.2019. 101199

9. R. Cheung, T. Ng, J. Wong, W. Chan, Chitosan: an update on potential biomedical and pharmaceutical applications. Mar Drugs 13，5156-5186 (2015). https://doi.org/10.3390/md 13085156

10. M. Prabaharan, Review paper: chitosan derivatives as promising materials for controlled drug delivery. J. Biomater. Appl. 23, 5-36 (2008)

11. Y. Cheaburu-Yilmaz, B. Kose, Chitosan-Graft-Poly(N-Isopropylacrylamide)/PVA cryogels as carriers for mucosal delivery of voriconazole. Polymers (Basel) 11, 1432 (2019). h ttps://doi.org/10.3390/polym11091432

12. Z. Naderi, J. Azizian, Synthesis and characterization of carboxymethyl chitosan/Fe3O4 and $\mathrm{MnFe} 2 \mathrm{O} 4$ nanocomposites hydrogels for loading and release of curcumin. J Photochem Photobiol B Biol 185, 206-214 (2018). https://doi.org/10.10 16/j.jphotobiol.2018.06.014

13. J. Huang, Q. Wang, L. Chu, Q. Xia, Liposome-chitosan hydrogel bead delivery system for the encapsulation of linseed oil and quercetin: Preparation and in vitro characterization studies. LWT 117, 108615 (2020). https://doi.org/10. 1016/j.lwt.2019.108615

14. Y. Qiu, K. Park, Environment-sensitive hydrogels for drug delivery. Adv. Drug Deliv. Rev. 53, 321-339 (2001)

15. J. Berger, M. Reist, J.M. Mayer et al., Structure and interactions in chitosan hydrogels formed by complexation or aggregation for biomedical applications. Eur. J. Pharm. Biopharm. 57, 35-52 (2004)

16. T.T. Yang, Y.Z. Cheng, M. Qin et al., Thermosensitive chitosan hydrogels containing polymeric microspheres for vaginal drug delivery. Biomed Res Int (2017). https://doi.org/ 10.1155/2017/3564060

17. K.A. Edwards, O.R. Bolduc, A.J. Baeumner, Miniaturized bioanalytical systems: Enhanced performance through liposomes. Curr. Opin. Chem. Biol. 16, 444-452 (2012)

18. K.A. Edwards, A.J. Baeumner, Liposomes in analyses. Talanta 68, 1421-1431 (2006)

19. T. Mahmoudi-Badiki, E. Alipour, H. Hamishehkar, S.M. Golabi, Dopamine-loaded liposome and its application in electrochemical DNA biosensor. J Biomater Appl 31, 273-282 (2016). https://doi.org/10.1177/0885328216650378

20. M. Alizadeh-Ghodsi, A. Zavari-Nematabad, H. Hamishehkar et al., Design and development of PCR-free highly sensitive electrochemical assay for detection of telomerase activity using Nano-based (liposomal) signal amplification platform. Biosens Bioelectron 80, 426-432 (2016). https://doi.org/10. 1016/j.bios.2016.01.090

21. J. Wang, Nanoparticle-based electrochemical bioassays of proteins. Electroanalysis 19, 769-776 (2007). https://doi.org/ 10.1002/elan.200603789

22. L. Liu, D. Deng, W. Sun et al., Electrochemical science electrochemical biosensors with electrocatalysts based on metallic nanomaterials as signal labels. Int J Electrochem Sci 13, 10496-10513 (2018). https://doi.org/10.20964/2018.11. 47

23. A. Zavari-Nematabad, M. Alizadeh-Ghodsi, H. Hamishehkar et al., Development of quantum-dot-encapsulated liposomebased optical nanobiosensor for detection of telomerase activity without target amplification. Anal Bioanal Chem 409, 1301-1310 (2017). https://doi.org/10.1007/s00216-016-0058$\mathrm{Z}$

24. K.Y. Chumbimuni-Torres, J. Wu, C. Clawson et al., Amplified potentiometric transduction of DNA hybridization using ion-loaded liposomes. Analyst 135, 1618-1623 (2010). http s://doi.org/10.1039/c0an00198h

25. T. Bortolamiol, P. Lukanov, A.M. Galibert et al., Doublewalled carbon nanotubes: Quantitative purification assessment, balance between purification and degradation and solution filling as an evidence of opening. Carbon N Y 78, 79-90 (2014). https://doi.org/10.1016/J.CARBON.2014.06. 051

26. D.R. Chen, P.K. Adusei, M. Chitranshi et al., Electrochemical activation to enhance the volumetric performance of carbon nanotube electrodes. Appl Surf Sci 541, 148448 (2021). h ttps://doi.org/10.1016/J.APSUSC.2020.148448

27. S.K. Hassaninejad-Darzi, M. Rahimnejad, Electrocatalytic oxidation of methanol by ZSM-5 nanozeolite-modified carbon paste electrode in alkaline medium. J Iran Chem Soc 11, 1047-1056 (2014). https://doi.org/10.1007/s13738-013-03737

28. A. Shrivastava, Methods for the determination of limit of detection and limit of quantitation of the analytical methods. Chronicles Young Sci 2, 21-25 (2011). https://doi.org/10.41 03/2229-5186.79345

29. I.-H. Cho, J. Lee, J. Kim et al., Current technologies of electrochemical immunosensors: perspective on signal amplification. Sensors 18, 207 (2018). https://doi.org/10.339 0/s18010207

30. C. Fenzl, T. Hirsch, A.J. Baeumner, Nanomaterials as versatile tools for signal amplification in (bio)analytical applications. TrAC - Trends Anal. Chem. 79, 306-316 (2016)

31. M.S. Amini-Fazl, R. Mohammadi, K. Kheiri, 5-Fluorouracil loaded chitosan/polyacrylic acid/Fe 30 O 44 magnetic nanocomposite hydrogel as a potential anticancer drug 
delivery system. Int J Biol Macromol 132, 506-513 (2019). h ttps://doi.org/10.1016/j.ijbiomac.2019.04.005

32. R. Foroutan, S.J. Peighambardoust, R. Mohammadi et al., Influence of chitosan and magnetic iron nanoparticles on chromium adsorption behavior of natural clay: Adaptive neuro-fuzzy inference modeling. Int J Biol Macromol 151, 355-365 (2020). https://doi.org/10.1016/j.ijbiomac.2020.02. 202

33. S. Ge, Ultrasensitive electrochemical immunosensor for CA 15-3 using thionine-nanoporous gold-graphene as a platform and horseradish peroxidase-encapsulated liposomes as signal amplificationitle. Analyst 137, 4440-4447 (2012). https://doi. org/10.1039/C2AN35751H

34. A.B. Ganganboina, A.D. Chowdhury, I.M. Khoris et al., Dual modality sensor using liposome-based signal amplification technique for ultrasensitive norovirus detection. Biosens
Bioelectron 157, 112169 (2020). https://doi.org/10.1016/J.BI OS.2020.112169

35. H. Ye, K. Yang, J. Tao et al., An enzyme-free signal amplification technique for ultrasensitive colorimetric assay of disease biomarkers. ACS Nano 11, 2052-2059 (2017). http s://doi.org/10.1021/acsnano.6b08232

36. F. Ghorbanizamani, H. Moulahoum, F. Zihnioglu et al., Quantitative paper-based dot blot assay for spike protein detection using fuchsine dye-loaded polymersomes. Biosens Bioelectron 192, 113484 (2021). https://doi.org/10.1016/J.BI OS.2021.113484

Publisher's Note Springer Nature remains neutral with regard to jurisdictional claims in published maps and institutional affiliations. 